\title{
Advanced Characterization and Testing of Irradiated Materials
}

\author{
DHRITI BHATTACHARYYA $\mathbb{0},{ }^{1,2,6}$ FAN ZHANG ${ }^{3}$ and \\ PETER HOSEMANN ${ }^{4,5}$
}

\begin{abstract}
1.-Australia's Nuclear Science and Technology Organisation, New Illawarra Road, Lucas Heights, NSW 2234, Australia. 2.-School of Materials Science and Engineering, UNSW, Sydney, NSW 2052, Australia. 3.-Materials Measurement Science Division, National Institute of Standards and Technology, Gaithersburg, MD 20899, USA. 4.-Department of Nuclear Engineering, University of California, Berkeley, CA 94720, USA. 5.-Material Science Division, Lawrence Berkeley National Laboratory, Berkeley, CA 94720, USA. 6.—e-mail: dhriti1@gmail.com
\end{abstract}

The rising global demand for energy due to increasing population is putting considerable pressure on the existing resources of conventional fuels, such as coal, oil, and natural gas. In the modern economy, energy consumption is coupled to economic growth due to its dependence on industrial production. While the electrical needs of Organisation for Economic Co-operation and Development (OECD) countries are increasing annually at $0.2 \%$, for non-OECD countries this rate is about $4.6 \%$. The incessant burning of fossil fuels to meet these demands has increased the emission of greenhouse gases. As a result, the current atmospheric $\mathrm{CO}_{2}$ concentration is above $400 \mathrm{ppm}$-its highest level since the dawn of human civilization.

These two phenomena have prompted the search for viable alternative sources to satisfy burgeoning demand without causing further damage to the environment. While renewable energy sources such as solar, wind, hydroelectric power, etc. are attractive for their low environmental impact, they are both unreliable and insufficient in their energy yield, at least at their present stage of technological development, as these sources are largely tied to geography and local weather conditions. On the other hand, nuclear energy is independent of any external influence with little need for power storage, making it an excellent choice for base load production.

The current global electrical energy demands are met with a mix of coal (38.3\%), natural gas (22.9\%), hydro (16.3\%), nuclear (10\%), and other renewables (8.5\%), according to the 2018 International Energy Agency study. Interestingly, in OECD countries, the share of nuclear power generation is $17 \%$ and that

Dhriti Bhattacharyya, Fan Zhang and Peter Hosemann are Guest Editors for the TMS Advanced Characterization, Testing, and Simulation Committee and Nuclear Materials Committee. of coal is $25 \%$, indicating that a large fraction of the coal-generated electricity is replaceable by nuclear power. It is remarkable that, in spite of a reduced number of reactors operating today, the same power output can be achieved due to continuous innovation in materials and design.

However, the current fleet of reactors is not without challenges, such as the safe storage or reuse of the highly radioactive nuclear waste they produce as well as accident concerns, public perceptions of risk, and fear.

The approaches that would help the increasing energy needs to be met more safely through the use of nuclear reactors include increasing their fuel efficiency (e.g., by operating at high temperatures), using beneficial breed and burn ratios to minimize nuclear waste while producing more fuel, improving the properties of the structural materials, and so on. These steps help to increase energy production and simultaneously reduce the amount of radioactive waste released into the environment. While most nuclear power is generated via conventional light water reactors, several other types of reactors have been constructed in the past and operated continuously over the years, such as the fast flux test facility (FFTF) or Bor 60 . The next generation of nuclear reactors, called Gen IV reactors [e.g., very high temperature reactors (VHTRs) and molten salt reactors (MSRs)], are adopting these and other strategies to achieve high yield and increased safety with minimal environmental impact.

In general, the structural materials of the core and surrounding areas of the reactors are subjected to high radiation doses and temperatures, which cause damage to their microstructure and result in hardening and embrittlement. Gen IV reactors will be operating under even more extreme conditions of temperature (up to $\sim 1000^{\circ} \mathrm{C}$ ), radiation dose (up to $\sim 200$ displacements per atom, dpa), and corrosive 
environments (such as molten salts), sometimes being subjected to them simultaneously. This situation therefore demands a thorough understanding of the effects of these conditions on material microstructure and properties, and the design and manufacture of materials which can withstand such extreme conditions. These scientific and technological demands necessitate the development of techniques to characterize and test irradiated materials in greater detail and higher efficiency. This special issue, therefore, aims to bring together some of the latest and most interesting studies in advanced characterization and testing of irradiated materials.

One of the central problems when studying the effects of neutron irradiation is that sample irradiation is a very slow process; it might take years or decades to attain a substantial dose in a conventional reactor. Moreover, irradiation by neutrons makes the samples radioactive, which necessitates the storage of the samples in "cooling pools" for extended periods of time before they can be handled. Even after prolonged storage to reduce the sample radioactivity, expensive "hot cells" may be necessary for the handling and testing of these samples. For these reasons, the use of high-energy ion irradiation to simulate neutron damage in materials has become increasingly popular in the past decades. However, this technique, while being faster than neutron irradiation by several orders of magnitude, creates shallow layers of damaged material on the sample surface with steep gradients in the damage profile. This makes it challenging to characterize and test the irradiated volume. Recent developments in focused ion beam (FIB) techniques have made it possible to extract samples for transmission electron microscopy (TEM) and fabricate specimens for micromechanical testing from such small volumes. This has opened new opportunities for exploring the effects of ion irradiation on materials. In the article "Towards Bridging the Experimental Length-Scale Gap for Tensile Tests on Structural Materials," Ajantiwalay et al. explore issues in microscale testing with respect to mesoand macroscale testing. There are quite a few interesting papers exploring the application of the small-scale testing methods for ion-irradiated materials, some of which are relatively new. These include the paper by Sadeghilaridjani et al., titled "Small Scale Mechanical Behavior of Ion Irradiated Bulk Metallic Glass," where the authors show that bulk metallic glasses are softened by ion irradiation, which makes them attractive for nuclear applications. Another paper by Moschetti et al, "On the Room-Temperature Mechanical Properties of an Ion-Irradiated TiZrNbHfTa Refractory High Entropy Alloy," explores through in situ microtensile testing the interesting mechanical properties of a body-centered cubic (BCC) high-entropy alloy after ion irradiation, and demonstrates the use of the technique in measuring consistently the mechanical property changes at these length scales, i.e., $\sim 1$ micron to 10 microns, especially when the grain size is small (tens of nanometers).

Another relatively easy and rapid method of assessing the mechanical properties of ion-irradiated materials is instrumented nanoindentation, which has been very popular in the community for more than a decade. Although the interpretation of the results is complicated due to the three-dimensional stress state around a nanoindent and its complex interaction with the ion-irradiated volume of material with a steep damage gradient, it provides a quick way to assess the mechanical property changes in a reliable, albeit semiquantitative manner. The paper "High Temperature Nanoindentation of SiC/SiC Composites" by Frazer et al. utilizes this technique to assess the mechanical properties of a $\mathrm{SiC}$ fiber/matrix composite, which is attracting increasing attention as a potential candidate for current and Gen IV nuclear reactors.

The characterization of microstructural changes due to irradiation, such as defect clusters, inert gas bubbles, dislocation loops, stacking fault tetrahedra, etc., is of paramount importance as they are the cause for the various mechanical property changes due to irradiation. A number of papers in this special issue deal with microstructural characterization of irradiated material through TEM performed on FIB-extracted samples, which enables the user to obtain samples from small volumes and/ or highly precise areas of interest. These include the paper by Derby et al, "Faceted He-Filled "Pancakes" Confined within Nano-Scale Metal Layers," where an interesting morphology of He bubbles present in a multilayered structure is studied. The paper by Hoffman et al, "Effects of $\mathrm{Al}$ and Ti Additions on the Irradiation Behavior of an FeMnNiCr Multi-Principal Element Alloy" uses the FIB-TEM technique to characterize the differences in irradiation-induced loop sizes between the two types of the alloy investigated, with and without $\mathrm{Al}$ and $\mathrm{Ti}$ additions. They also use nanoindentation to show differences in irradiation-induced hardening at two irradiation temperatures.

The role of twin boundaries as sinks for radiation damage in nanotwinned metals is explored in an expansive review by Yu et al., titled "Recent Studies on the Microstructural Response of Nanotwinned Metals to In Situ Heavy-Ion Irradiation." In this paper, the authors provide an overview of recent studies on the microstructural response of nanotwinned metals to in situ heavy-ion irradiation, with an emphasis on the "interactions of defect clusters with twin boundaries and radiation induced twin boundary migration."

The implantation of $\mathrm{He}$ in materials generally causes the formation of He-filled bubbles and cavities, which can cause macroscopic dimensional changes referred to as "swelling." The swelling in He-irradiated $\mathrm{SiC}$ fiber/ $\mathrm{SiC}$ matrix composites was 
characterized by an interesting atomic force microscopy (AFM) technique to show their differential expansion in the paper "Localized Helium Implantation in $\mathrm{SiCf} / \mathrm{SiCm}$ Composites Comparing Fiber and Matrix Swelling" by Ambat et al.

He bubbles in metals can also be characterized by diffraction experiments, and the paper by Hammons et al, entitled "He Bubble Concentration, Size and Strain in Implanted Aluminum by SAXS/WAXS" uses these techniques to delve deeper and try to estimate the fraction of $\mathrm{He}$ inside the bubbles, and that remaining as defects in the crystal. It also shows that the fraction of $\mathrm{He}$ in the bubbles is dependent on the incident He-ion beam energy.

Continuing with diffraction methods, the paper by Vogel et al, "Advanced Post-irradiation Characterization of Nuclear Fuels Using Pulsed Neutrons" explores a new method of characterizing irradiated fuel materials in the bulk through pulsed neutrons.
Last but not the least, this issue includes an extremely interesting paper by Dennett et al. titled "Listening to Radiation Damage In Situ: Passive and Active Acoustic Techniques," which demonstrates the use of acoustic emission and transient grating spectroscopy to detect the onset of damage events in quartz and $\mathrm{Pd}$ and the formation of small defect clusters in self-ion-irradiated $\mathrm{Cu}$, respectively. This paper should help in broadening the use of these techniques for in situ characterization of radiation damage in ion-beam experiments.

These articles are published under the topic "Advanced Characterization and Testing of Irradiated Materials" in the January 2020 issue (vol. 72, no. 1) of $J O M$, and can be accessed at http://ink. springer.com/journal/11837/72/1/page/1.

Publisher's Note Springer Nature remains neutral with regard to jurisdictional claims in published maps and institutional affiliations. 